\title{
Digital Re-Evolution: Learning to be Human at Contemporary Society
}

\author{
Aita Sakun \\ Doctor of Philosophical Sciences, Associate Professor, Kyiv National University of \\ Technologies and Design (Kyiv, Ukraine) \\ E-mail: ayta.s@ukr.net \\ https://orcid.org/0000-0002-7889-2358
}

\section{Irina Mordous}

Ph.D. in Pedagogy, Associate Professor, National Pedagogical Dragomanov University (Kyiv, Ukraine)

E-mail: i.a.mordous@gmail.com

https://orcid.org/0000-0003-1659-001X

\begin{abstract}
The problem of human adaptation to living conditions in the society of the digital revolution is the main content of this study. The change of the communicative paradigm, the essence of social relations and interactions, the problem of critical thinking and the humanitarian basis of classical education is analyzed through the prism of the problem of socio-cultural transformations, social justice, and identification procedures of the modern individual. The article develops the key problem of The XXIV World Congress of Philosophy through the analysis of educational mechanisms of prevention of human nature and spiritual orientations in the face of digital era challenges.

Keywords: digitalization; Digital Earth; new social paradigm; advanced-learning technologies; communication; virtualization; visualization
\end{abstract}

Received: September 12, 2018; accepted: October 10, 2018

Philosophy and Cosmology, Volume 22, 2019: 129-136.

https://doi.org/10.29202/phil-cosm/22/11

\section{Introduction}

Modern society has traditionally received a predication as information. The meaning of this transformation is to change not only the way of production and distribution of material and spiritual goods, but also a more fundamental shift to a qualitatively new way of explication of the socio-cultural essence of man. Often this transformation is associated with the computerization of society. This connection is not accidental, because the introduction

\footnotetext{
(C) Sakun, Aita, 2019
}

(C) Mordous, Irina, 2019 
of digital technologies in all spheres of social life radically changes the nature of socioeconomic organization, the relevant strategies of education and the formation of human nature. It is natural that in society, the dynamics of which predetermines the phenomenon of innovation, interpretation techniques with respect to the content and role of education change significantly. Rapid changes in the socio-cultural context of the horizon of human existence actualize the question of how to determine the human nature. Accordingly, one of the leading themes of The XXIV World Congress of Philosophy was the problem of relearning-to-be-human. Therefore, the question of the General logic of transformation of the modern socio-cultural context and, accordingly, ways of adaptation to it, both natural and systemic, is extremely relevant for modern Humanities.

\section{Social Justice and Social Stability at Digital Era}

Modern society postulates the principles of democracy and liberalism as fundamental values. It is possible to implement them in public practice only on condition of an equal and fair order of organization of public life. The problem is that at the present stage, possession of information, as well as free access to it, is a criterion of openness and liberalism of society. It is this defining fact that Yu Cheung Wong and his co-authors focus on, noting: "Digital inclusion references to the extent to which the excluded segments of a society can have equal opportunity to take part in the information society. Promoting digital inclusion aims to close such opportunity gaps. The premise for having an opportunity to take part in the information society is having access to information and communication technology. The degree to which disabled people actually use these tools to engage in the information society also reflects the extent to which equal opportunity exists. Conceptually, accessibility and actual use are interwoven and serve as important indicators of whether disabled groups have equal opportunity to take part in the information society" [Yu, 2009: 61]. Issues of social stratification are of particular importance in the information society and the ways of human life receive new forms of determination in the information society.

The problems of equality and social stratification naturally lead to the articulation of the question of the realization of fundamental human rights. Kay Mathiesen formulates his theoretical and methodological vision of the problem: "I do not propose a list of digital rights here; rather, I want to suggest how we might approach developing such a list. There are a number of questions we can ask ourselves that would help us to formulate a declaration of digital human rights: for any particular right, what would it mean to respect this right in the digital realm? What new rights we might need to articulate to address the unique context of the digital realm? What rights may not transfer to the digital realm at all? Are there some rights that are becoming irrelevant given the digital and communications revolutions?" [Mathiesen, 2014: 7]. In the course of artificial intelligence, the author produces a list of new questions arising in the study of this problem, namely: "In answer the question, "What would it mean to satisfy a particular human right in the digital realm?" it will help to consider a series of further questions: 1 . What is the right and what competing interest does it protect? 2 . How does this right support other rights? 3. What other rights must it be balanced against? 4 . What new opportunities do information technologies create for satisfying this right? 5 . What new threats do information technologies pose to this right? 6 . What institutional arrangements might need to be changed or put in place to respect, protect, and fulfill this right in the digital context?" [Mathiesen, 2014: 8]. The whole range of these questions outlines the fundamental motives of human existence. 
Indeed, in the history of thought, man is traditionally understood as spiritual integrity, not actual, but potential in nature. From a set of mental, physical, spiritual, moral inclinations of the person during socialization the unique complex phenomenon of the personality is formed. The contradiction of essence and existence, ontologically separated, get the integrity of unity in the process of human life in society. Accordingly, the freedom of creativity and human activity design socialized uniqueness of the individual. In the information society, the factors influencing the mechanisms of freedom and responsibility are much greater than in the traditional or industrial society. Intensive communication links, in which the course of everyday life takes place, attract a person to the large-scale sphere of information flows, orientation and interaction of which cause many threats in terms of information security and personal integrity. The digital era came to social being together with 'mobility turn', which causes "...transformation of ideas about space and time at a contemporary society, which undergoes the radical changes to the established principles of social interaction" [Savenkova \& Svyrydenko, 2018: 59].

The processes of unification and globalization of the modern world require appropriate technical and technological support. The answer to such challenges of modern socio-culture is the Digital Earth project, which includes multi-component techniques for optimizing communication processes. And the special significance of these projects lies in the fact that at the present stage of development of technologies and civilization, they acquire signs of strategies for the survival of mankind. Therefore, Timothy W. Foresman formulates his own vision for the issue: "As long as the community can keep open and free access to the major of data generated from government sponsored sources, Digital Earth 3D geobrowsers can be seen to become a common and ubiquitous tool for all citizens as they wrestle with the daily challenges of their lives. Citizens around the globe are beginning to forge organized progress towards sustainable solutions, as copying strategies, for their future and their children's future, especially as the specter of climate change becomes more evident from science and personal observations. This new computerised nervous system of the planet will provide a force proactive for the betterment of the human species and the other living resources of this planet. It will be incident upon the information community to help ensure that the future of Digital Earth and 3D geobrowsers services all humankind" [Foresman, 2007: 15]. Indeed, access to information and the competence to assimilate and apply it in the modern age is as much a necessary resource for survival as the factors of congratulatory variety. The General indicator of the impact of mankind on the natural environment is qualitatively high, so it is reasonable to believe that the human habitat is above-natural. Indeed, the cultural horizon produces a corresponding dimension of virtual existence, the digital dimension of reality as an explication of the potential essence of man. Thus, from a variety of projects of possible future, a person is able to create a chosen version of the natural development of events. Mario Hernandez says in detail about this problem: "Future Earth aims to focus on answering fundamental questions such as how and why the global environment is changing. To predict and model future changes? It will undertake research to provide scientific-based options aimed to reduce losses caused by natural disasters. The research is oriented to identify ways to implement transformations to prosperous and equitable futures" [Hernandez, 2017: 2]. Continuing the author's idea, we note that the creation of such a digital project is an effective means of increasing the reflexivity of perception and self-identification of the General public in the context of rapid changes in the eventful daily life. Interaction of subjects of activity of different levels of the organization: from individual, public, state and international, requires a systematic and competence-based approach to the establishment of communicative 
discourse. Therefore, naturally, "Both, Future Earth and Digital Earth, are emerging under the political Agenda 2030, Agenda that aims to achieve overall sustainability and recognizes and requests the need to have scientific evidence supporting the decision making process. Future Earth, jointly with many other institutions, is setting up the scientific research platform, while Digital Earth is setting up the tools where such scientific information could be derived. While the political agenda provides an excellent atmosphere for Future Earth and Digital Earth to succeed, the main challenge is for both activities to do so" [Hernandez, 2017: 3]. Therefore, these strategies for creating digital projects of international and international communication, virtual visibility of the achievements of human civilization have reason to be considered as a global initiative to integrate efforts to consolidate humanity in the face of possible challenges of natural, social, political, man-made varieties. This perspective of the problem is quite natural. After all, communication is essentially a semiotic problem, as its effectiveness depends on the chosen means and techniques of definition. Projects related to Digital Earth are aimed at establishing communication through visualization of ideas and knowledge about the world. Visual images are devoid of T. V. translation difficulties, and the speech facilities used by these projects perform a supporting function in the communication. This method of objectification of knowledge preserves the semantics and pragmatics of the content of information, but eliminates the meaning of syntax, and hence the procedures for demonstrating the thesis, its rationale and significance. Given the global growth of quantitative indicators of information in modern culture and the intensification of the pace of social life, visualization as a way of representing knowledge should be considered as a necessary companion of fundamental ideological shifts. Indeed, not only individual natural languages have a potentially defined set of lexical and grammatical structures of utterance. A separate historical era produces a unique episteme or discourse, according to Michael Foucault, or a separate model of scientific research, a paradigm in the terminology of Thomas Kuhn. Even certain segments of scientific knowledge operate with their own thesaurus, which potentially makes professional communication impossible. Michela Massimi gives the following example: "There are two main assumptions underpinning the untranslatability thesis. The first is the identification of lexical structures with lexical taxonomies. So, for example, the Aristotelian statement 'free fall is accelerated motion' cannot be translated into the Galilean lexicon, because although the term 'free fall' appears as a kind term in both lexicons, overlap the two without the sentence being translatable, because a major conceptual change has in the meanwhile occurred (e.g. from free fall as a force-free motion towards a natural place within the Aristotelian lexicon, to forced motion within the Galilean lexicon). A second important assumption is that taxonomy must be preserved for translation to be possible. But the nooverlap principle makes taxonomy preservation impossible, by barring overlapping between taxonomic categories relating to the same contrast set (while allowing inclusive overlapping of genusespecies type)" [Massimi, 2015: 87]. Therefore, it is absolutely appropriate to think about the need to develop methods for visualizing the space-time model of human knowledge about the near world, about the planet. This model organically combines individual objects and processes into a balanced dynamic system. Accordingly, a new way of organizing communication, objectification of knowledge and the formation of subjectivity are necessary prerequisites and factors of the digital revolution.

The digital revolution, which has actually occurred in the modern world, permeates all spheres of human life. The introduction of automation, computerization and data processing technologies not only accelerates the course of social, economic, political and educational processes, but also changes their content. Politics is transformed into the sphere 
of representation, sociality is characterized by the anonymity of the network model, and the economy is reoriented from production to service and marketing. Therefore, it is logical that digitalization leads to a change in the communicative paradigm of society. Digitalization is not just a new technique of representation and translation of content, but a comprehensive solution to a range of managerial, logistical, behavioral problems. Instead of the idea of the fundamental nature of knowledge, which exposes the description of the events of reality to the post-fact, digitalization is the realization of the volitional principle, the principle of liberalism, which does not describe, but States and projects reality. An example of such explication of human willpower is such a product of modern social culture as Megatrends. This notion is adjacent not only to the economic dimension. General trends in global geopolitics, the idea of standards of social life and the principles of a just society, educational standards and advanced algorithms of scientific research - all these areas of modern life are regulated by beliefs that have every reason to be considered metatrends. This approach to the understanding of reality as a plastic to the will and ambitions of a man lies in understanding how the future world of new possibilities, the horizon of realization of which is the scene of the approbation of your wildest dreams.

Thus, a person creates around him an intellectualized space of things and structures that the complexity of the organization is dominated by the possibility of knowing the average man in the street. However, comfortable living conditions, as you know, slow down, if not inhibit the formation of adaptive mechanisms of the individual. Development is possible only as overcoming and struggle, or going beyond the comfort zone. The absence of such a struggle for survival has a corresponding impact on the course of evolutionary processes of adaptation to the environment. Therefore, there is a threat of a demarcation gap in the level of education and culture between different representatives of the human community. Against the background of modern globalization processes and close communication links, this problem is already quite obvious. We consider the so-called migration crisis, which affects countries with developed economies and high social standards, to be a confirmation of its scale and significance. After all, even if there is a positive progressive historical development of civilization, to expect its effectiveness within a single human life is a utopia. Therefore, individuals-carriers of archaic systems of values and stereotypes pose a threat to the stability of the existence of the countries to which they aspire for a better life. The reason probably is that get what you want (social protection and satisfaction of vital needs) subjectivity is difficult to shift to new, but advanced culture, patterns and values. All these trends are controversial from the standpoint of stabilization of public life. However, the socalled "intellectual explosion concept" also represents another aspect of possible threats. We are talking about the possible risks of creating artificial intelligence and a riot of machines. If machines and automated systems are created to meet human needs, then the notion of their self-sufficient mode of existence without attribution to human is certainly alarming. Perhaps the creation of Digital Earth projects is an attempt to organize the chaotic processes synergetic on the grounds of reality, and the practice of focusing for the implementation of analytical and reflective activities.

\section{Transformation of Education in the Digital Age}

Taking into account the fundamental changes in the social culture of modernity, the content of educational strategies changes radically. Digitalization eliminates the understanding of the role of the teacher as a carrier of information, because the information is available to the General public. The teacher is not the one who reports, but the one who 
forms the competence of navigation in information flows. Digitalization does not imply the acquisition of knowledge, but the acquisition of competencies that are subject to permanent correction depending on the development and implementation of innovative technologies. Wide access to information is a solid base of potentiality, to teach how to transform it into actual achievements of a person-this is the task of a modern teacher. And the main problem, given the above, is not even the methodology of training, and the methodology of motivational impact. So imagine the educational technology digital days without significant humanitarian component, reckless and dangerous. Especially given the close infrastructure ties of the modern world, the tragedy of world terrorism, the colonialist and Imperial ambitions, the problem of educational activities is of particular importance and urgency. Especially among the younger generation. For example, Sandy Schuck and Peter Aubusson note: "A serious appreciation of the cultures, contributions, needs and characters of young people requires a deep understanding of adolescents' current and emerging online practices, their benefits and pitfalls, their implications for formal education, and the development of guidelines for the management and uptake of social technologies in Association schools. The potential for digital technologies to contribute to a useful, productive and engaged citizenship seems significant" [Schuck \& Aubusson, 2010: 302]. The Digitalization of education, both technically and content-wise, necessarily implies a human-anthropological basis. Only under such conditions is it possible to overcome the modern transitive nature of planetary society. After all, our planet is divided not only by time zones and climatic zones, but also by various cultural axiology and epochs of historical time. It is possible to coordinate the rapid growth of technologies with the unlimited needs of mankind and the limited resources of the planet provided the formation of individual learning routes, high standards of self-motivation and reflection, the involvement of virtual and augmented reality technologies. Depending on the tasks set before the formation of plasticity and variability, acquire its components such as the schedule and rate of assimilation of the program, the choice of forms and methods of training, teacher or tutor, and so on.

Digitalization of education provides for the continuity of the educational process, so widely implemented technology life-long-learning - lifelong learning and advancedlearning technologies - individualization of learning advanced level of knowledge and competencies. These phenomena are absolutely natural, given the era of accelerating technological changes, a significant change in professions in the labor market. Thus, the shelf life of the competence is constantly reduced, and of course, much less than the life of an individual specialist. Therefore, the classical education system, which remains inertial and reactionary, is not able to meet the needs of the modern market of developed capitalism. The existing gap between education, science and production in the modern world is the cause of many problematic situations. It is natural that operational solutions to problem situations are possible in operational systems, among which the education system does not apply. The contradiction in this case lies in the contextuality of effective solutions to the operationality and universality of classical education. The optimal solution will be a combination of these approaches to the implementation of educational services: the universality of the classics and the efficiency of individual research projects. The question is how they are mutually organized and how to find the degree of interaction.

\section{Digitalization' Challenges for Human Nature}

The digitalization of the modern era radically changes the identification procedures of human nature. For example, Fiona Chambers and Rachel Sandford argue: "We argue that an 
uncritical appreciation of the acquisition and transfer of social-technical capital between and across such spaces holds significant implications for young people's construction of identity and associated digital well-being. We further assert that PE, with its pro-social values focus, is a key site for empowering young people to become critical prosumers [simultaneously producing and consuming] of knowledge and social capital and thus to flourish i.e. enjoy digital wellbeing" [Chambers \& Sandford, 2018: 5]. Without a classical education-based humanitarian consciousness orientation in the digital environment threatening two opposing trends: consumerism and aggressiveness. It is no coincidence that the areas in which the most rapidly developing technology of virtual and augmented reality, is the sphere of entertainment and military-industrial complex. Therefore, the question of motivation and reflection of the dynamics of self-activity not only did not disappear from the research focus in the digital age, but rather, make it main-stream: “...in a world where educational culture is heavily influenced by technological change, it is imperative for researchers (and practitioners) to make reflective spaces in which such issues can be considered. We assert that supporting the development of each pupil's Personal Values Compass can provide this space and, Rover, that with such a resource at their disposal, pupils can be empowered to flourish and drive as humans in a digital world" [Chambers \& Sandford, 2018: 11].

Problems of humanity, ways of its interpretation and verification in the digital age are the main criterion in the implementation of navigation practices. According to Arjun Sabharwal, the digitalization of the achievements of humanitarian culture takes place in different ways (DH - digital humanities): "Developing from the field of humanities computing, DH embrace a variety of topics, from curating online collections to data mining large cultural data sets. DH currently incorporate both digitized and born-digital materials and combine the methods from traditional humanities disciples (such as history, philosophy, linguistics, literature, art, archeology, music, and cultural studies and social sciences with tools provided by computing (such as data visualization [sic], information retrieval, data mining, statistics, text mining) and digital publishing"[Sabharwal, 2017:3]. At the article, we stand of position, that interpretation, documentation and implementation in the assimilation of humanitarian knowledge are integral components of the formation of critical thinking. Note that for critical thinking as one of the leading and popular professional competencies of our time is characterized by the trampling of authorities. Therefore, the role of an intermediary in obtaining knowledge or access to information is gradually losing its importance and relevance in modern realities. Liberalism and voluntarism find their realization in such aspects of sociocultural transformations.

\section{Conclusions}

Man is an active being, and the digitalization of reality produces a fundamentally new way of adaptation practices. The effectiveness of this strategy should be ensured by the education system, which is also undergoing a puzzling transformation. The main attribute of modern man is communication as a way of existence in the information layers of modern culture. The nature of communication also varies from semantic-syntactic semiotics to visualization of intelligent constructs. This transition is explained by the need to intensify the communicative space in all aspects of life: political, social, educational, scientific, etc. It is indicative of voluntarism and liberalism of the digital age, which determines the overall dynamics of the processes in these areas. Thus, the biological evolution of mankind at a certain stage has acquired the features of sociality. Modernity produces grounds for verification of evolutionary mechanisms taking into account the digitalization of the socio-cultural sphere. 
Indeed, man is a product of historical development. Therefore, we consider it reasonable that it is the humanitarian culture that contains the necessary potential for effective adaptation and stabilization of public life.

\section{미] References}

Andreotti, Vanessa de Oliveira, and Karen Pashby. Digital Democracy and Global Citizenship Education: Mutually Compatible or Mutually Complicit? The Educational Forum, Vol. 77, Issue 4, 2013: 422-437. https://doi.org/10.1080/00131725.2013.822043

Chambers, Fiona, and Rachel Sandford. Learning to be human in a digital world: a model of values fluency education for physical education. Sport Studies and Physical Education, Vol. 1. Issue 1, 2018: 1-12. https://doi.org/10.1080/13573322.2018.1515071

Foresman, Timothy W. Evolution and implementation of the Digital Earth vision, technology and society. International Journal of Digital Earth, Vol. 1, Issue 1, 2007: 4-16. https:// doi.org/10.1080/17538940701782502

Hernandez, Mario. A digital earth platform for sustainability. International Journal of Digital Earth, Vol. 10. Issue 4, 2017: 342-355. https://doi.org/10.1080/17538947.2016.1272 646

Massimi, Michela. Working in a new world': Kuhn, constructivism, and mind-dependence. Studies in History and Philosophy of Science, Vol. 50. Issue 1, 2015: 83-89. https:// doi.org/10.1016/j.shpsa.2014.09.011

Mathiesen, Kay. Human Rights for the Digital Age. Journal of Mass Media Ethics: Exploring Questions of Media Morality, Vol. 29, Issue 1, 2014: 2-18. https://doi.org/10.1080/08 900523.2014 .863124

Sabharwal, Arjun. Digital humanities and the emerging framework for digital curation. College \& Undergraduate Libraries, Vol. 24, Issue 2-4, 2017: 238-256. https://doi.or $\mathrm{g} / 10.1080 / 10691316.2017 .1336953$

Savenkova, Liudmyla and Denys Svyrydenko. Academic Mobility and Academic Migration Issues: the Case of Ukrainian Higher Education. Interdisciplinary Studies of Complex Systems, No. 13, 2018: 57-65.

Schuck, Sandy and Peter Aubusson. Educational scenarios for digital futures. Learning, Media and Technology, Vol. 35, Issue 3, 2010: 293-305. https://doi.org/10.1080/174 39884.2010.509351

Yu, Cheung Wong, Chi Kwong Law, John Yat Chu Fung and Jolie Chi Yee Lam. Perpetuating Old Exclusions and Producing New Ones: Digital Exclusion in an Information Society. Journal of Technology in Human Services, Vol. 27, Issue 1, 2009: 57-78. https://doi. org/10.1080/15228830802459135 